\title{
Endometriosis: a rare cause of small bowel obstruction
}

\author{
Samir A Khwaja, ${ }^{1}$ Rasheed Zakaria, ${ }^{2}$ Herman Anthony Carneiro, ${ }^{3}$ Haris A Khwaja ${ }^{4}$
}

\author{
${ }^{1}$ Royal London Hospital, London, UK; \\ 2Department of Neurosurgery, The Walton Centre NHS Foundation Trust, Liverpool, UK; \\ ${ }^{3}$ Department of Neurosurgery, National Hospital for Neurology and Neurosurgery, London, UK; \\ ${ }^{4}$ Gravitas Bariatric Unit, Bourne End, UK
}

Correspondence to Dr Herman Anthony Carneiro, herman.carneiro@doctors.org.uk

\begin{abstract}
Summary
Although endometriosis is a common condition in young women, symptomatic involvement of the small bowel is rare. The authors report the case of a 44-year-old lady initially thought to have irritable bowel syndrome who presented 1 month later with acute small bowel obstruction. A CT scan showed small bowel dilatation with a transition point in the ileum, but no distinct lesion. The patient had an exploratory laparotomy where an obstructing lesion in the terminal ileum and several enlarged mesenteric lymph nodes were identified. Consequently, a right hemicolectomy was performed. Pathology specimens showed multiple endometriotic foci in the bowel with stricturing of terminal ileum and appendiceal intussusception. This likely resulted in subocclusive episodes and intestinal obstruction. This case highlights the difficulty in establishing a preoperative diagnosis of endometriosis. Small bowel endometriosis should, therefore, be considered in the differential diagnosis of women of childbearing age who present with symptoms of obstruction.
\end{abstract}

\section{BACKGROUND}

Small bowel obstruction (SBO) is a common cause of hospitalisation, representing $20 \%$ of all surgical admissions for acute abdominal pain. ${ }^{1}$ Adhesions, strangulated herniae and metastatic malignancy account for the vast majority of cases of adult small bowel obstruction in Western Europe. ${ }^{2}$ Endometriosis is the presence of benign endometrial tissue in extra-uterine sites and affects up to $8 \%-15 \%$ of women. ${ }^{3}$ Although most commonly located within the pelvis, the incidence of bowel involvement is $3 \%-37 \%$, primarily in the rectum and sigmoid colon. ${ }^{4}$ Reports of symptomatic endometriosis of the small bowel are rare. We describe a case of endometriosis of the terminal ileum presenting with acute small bowel obstruction.

\section{CASE PRESENTATION}

A 44-year-old nulliparous premenopausal Caucasian woman presented to a general hospital with a 3 day history of worsening, colicky central abdominal pain associated with distension, bilious vomiting and diarrhoea. The patient reported a history of altered bowel habit with episodes of diarrhoea, reduced appetite and one stone of unintentional weight loss in the 6 months before her presenting. She had been investigated with oesophago-gastro-duodenoscopy (OGD), which was unremarkable, and colonoscopy, which identified prominent lymphoid follicles in the terminal ileum, biopsies of which showed no evidence of malignancy or inflammatory bowel disease. She was therefore discharged with a diagnosis of irritable bowel syndrome. At presentation, the patient was at day 2 of her menstrual cycle. Her menstrual periods were regular with no menorrhagia, dysmenorrhoea or dyspareunia reported. The patient reported being unable to conceive but had not been investigated any further for this. There was no other medical history of note and no abdominal surgeries or laparoscopies. She was not taking any medications and there was no family history of Crohn's disease or ulcerative colitis.

\section{INVESTIGATIONS}

At initial assessment the patient had normal pulse, blood pressure, respiratory rate and temperature. Abdominal examination revealed a moderately distended abdomen which was diffusely tender. Bowel sounds were hyperactive. Digital rectal examination was unremarkable. A supine abdominal radiograph taken at admission showed multiple dilated loops of small bowel situated centrally and no gas in the large bowel (figure 1). There was no free air under the diaphragm seen on an erect chest radiograph. Her renal function, liver enzymes, bilirubin and electrolytes on admission were within normal limits. Haemoglobin was low at $11.1 \mathrm{~g} / \mathrm{dl}$ with no other abnormalities. Stool microscopy and culture were negative for pathogens. Stool faecal occult blood testing was not performed.

The patient's clinical presentation was that of small bowel obstruction of unknown aetiology. The patient was managed with fluid resuscitation, kept nil by mouth and a nasogastric tube passed. Given that she had no previous abdominal surgery, there were no herniae on clinical examination and she was not in the right age group for gastrointestinal malignancy, a CT scan of the abdomen and pelvis was ordered to help elucidate the cause (figure 2). Her abdominal CT with contrast performed the day after admission demonstrated small bowel dilatation with a transition point in the ileum, but no distinct mass lesion. She remained clinically stable but as her symptoms did not resolve with conservative management a MRI was ordered to image the small bowel more clearly and identify a possible focal lesion (figure 3 ). This showed frank small bowel obstruction with an area of focal nodular eccentric mural thickening. The radiological diagnosis was that of a small bowel tumour or a fibrotic lesion. There was no evidence of pathological lymphadenopathy. The uterus and ovaries were unremarkable and the ovaries and adnexae within normal limits. An 
ultrasound scan performed to further characterise this lesion showed an area of soft nodular tissue within the small bowel loops, possibly giving rise to intermittent intussusception.

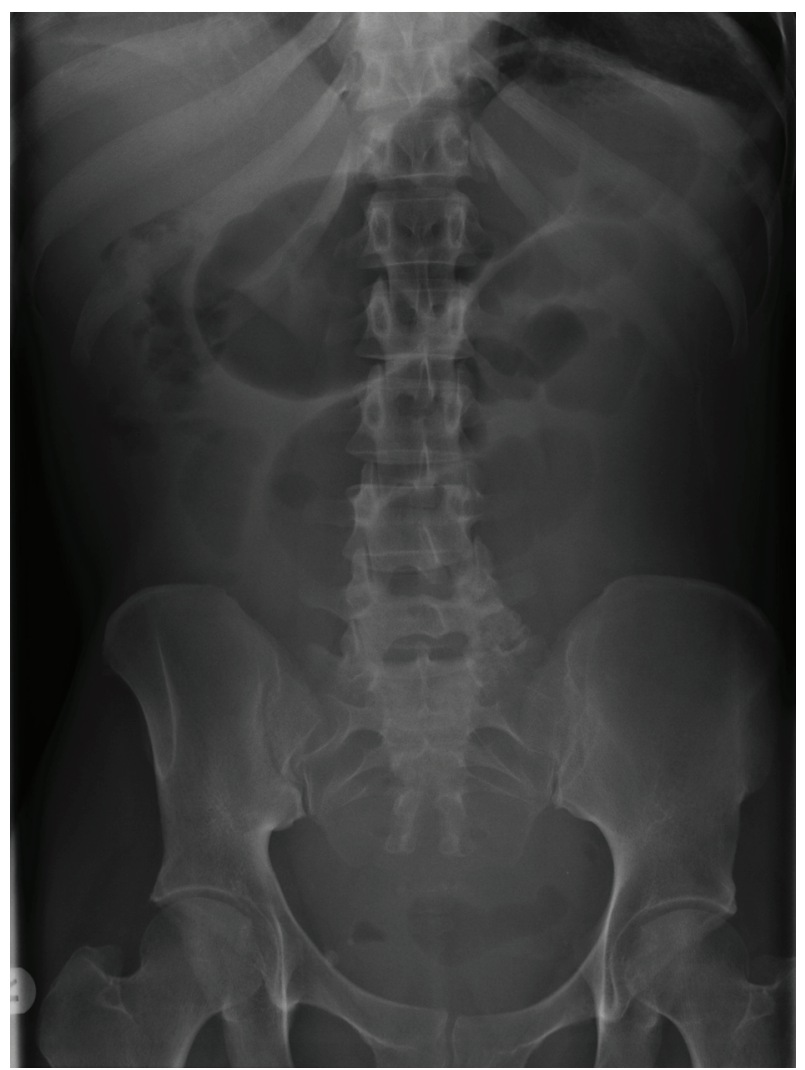

Figure 1 Erect abdominal radiograph showing multiple dilated loops of small bowel and the absence of gas in the colon.

\section{TREATMENT}

After further fluid resuscitation and nasogastric tube suction, an exploratory laparotomy was performed at which an obstructing lesion in the terminal ileum and several enlarged mesenteric lymph nodes were identified. A right hemicolectomy was performed with the enlarged lymph nodes included in the specimen. Subsequent histopathology confirmed endometriosis of the distal terminal ileum resulting in stricturing as well as endometriosis of the appendix resulting in intussusception. The changes within the mesenteric lymph nodes were reactive.

\section{OUTCOME AND FOLLOW-UP}

Postoperatively, the patient made an uneventful recovery and was discharged on day 6 . She was referred to a gynaecologist for further management of her endometriosis and will be followed up at 6 months by the general surgeons.

\section{DISCUSSION}

Endometriosis is a condition characterised by the presence of endometrial-like tissue outside the uterus. Although not particularly rare among women of childbearing age, establishing a preoperative diagnosis of intestinal endometriosis has proven a challenge. 6 The classical symptoms of endometriosis are infertility, dysmenorrhoea and dyspareunia. However, our patient first presented with colicky central abdominal pain, intermittent diarrhoea and constipation, weight loss and increasing abdominal distension over the previous 6 months. She was known to be infertile. Her initial investigations including OGD and colonoscopy were normal and she was diagnosed with irritable bowel syndrome. However, her condition did not improve and she presented 1 month later with colicky central abdominal pain, associated with distension, bilious vomiting and diarrhoea. These features suggested small bowel obstruc-

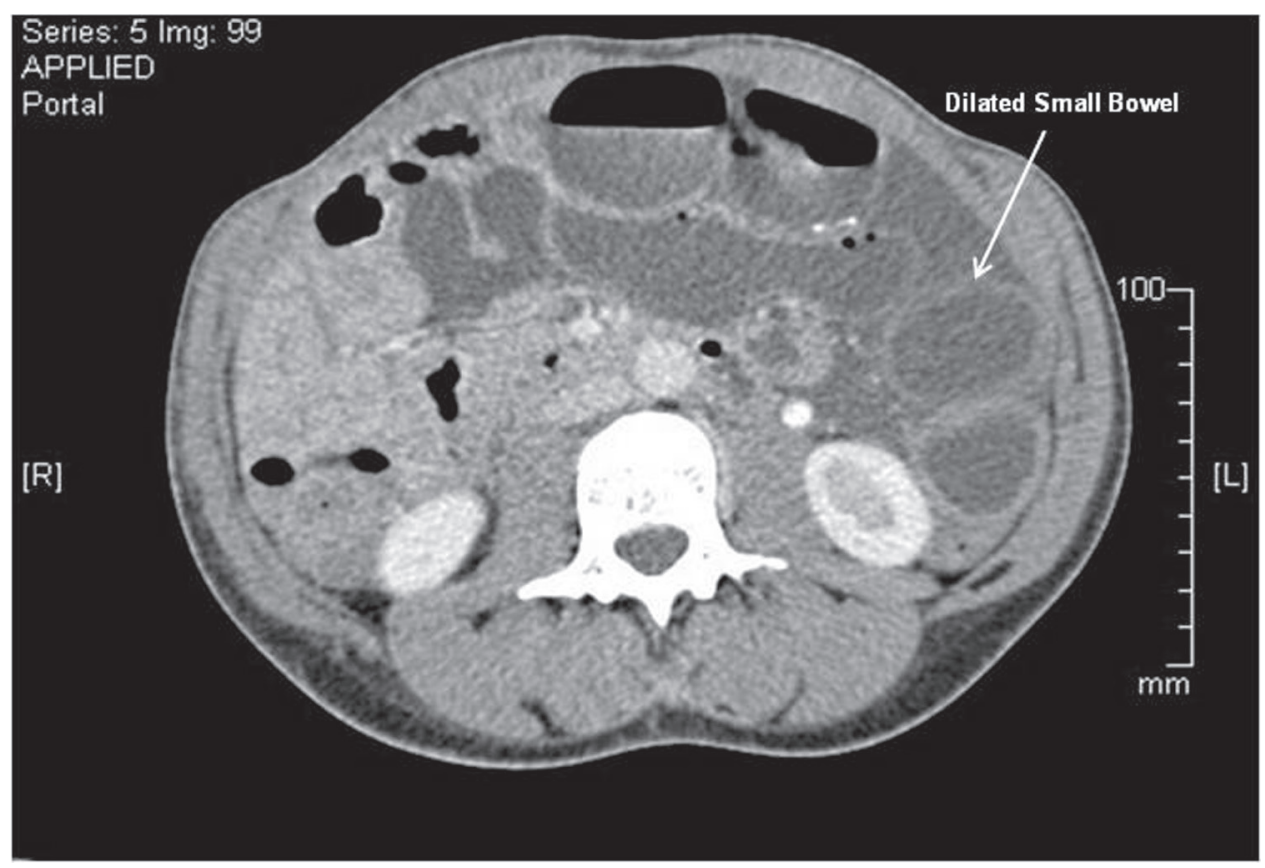

Figure 2 Abdominal CT scan image showing multiple fluid filled dilated loops of small bowel consistent with complete small bowel obstruction. 


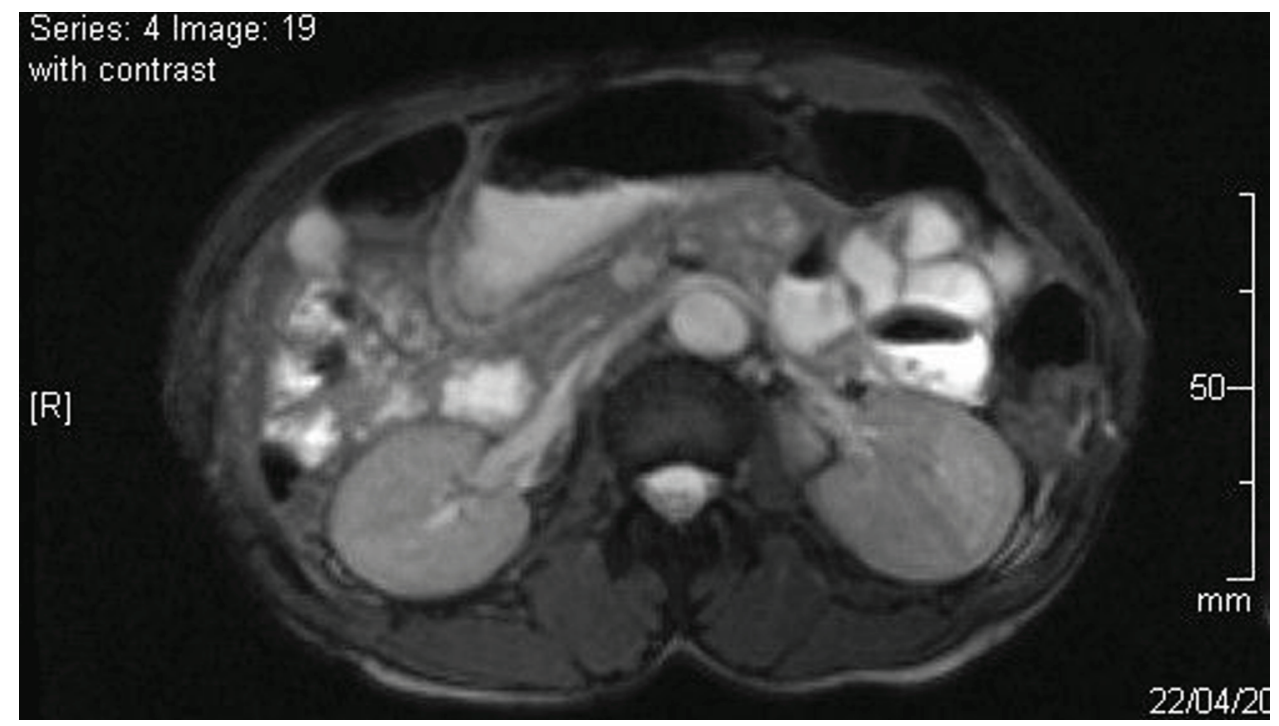

Figure $3 \mathrm{MRI}$ with contrast shows area of focal eccentric mural thickening, demonstrates only mild contrast enhancement.

tion, though establishing a diagnosis of ileal endometriosis proved to be more difficult.

The presence of endometriotic lesions in extrauterine locations such as the bowel is not unusual. A review of 7200 cases of endometriosis found only a $0.5 \%$ incidence (36 cases) of small bowel involvement. ${ }^{7}$ Most were found at surgery and only 11 patients $(0.15 \%)$ presented with small bowel obstruction. Establishing this diagnosis can be difficult as abdominal pain in women of reproductive age may mimic those of irritable bowel syndrome, infectious diseases, ischaemic enteritis/colitis, inflammatory bowel disease and malignancy. ${ }^{8}$ In our patient, her abdominal pain was non-specific, chronic and did not vary cyclically with the menstrual cycle. However, her secondary infertility and prior subocclusive episodes increased suspicion of ileal endometriosis. Endoscopic biopsies had not yielded this diagnosis; possibly as intestinal involvement is usually subserosal. ${ }^{5}$ Imaging was used to help establish a preoperative diagnosis. Radiological findings showed small bowel obstruction, however no distinct mass lesion was identifiable. In such cases MRI is usually helpful. In a typical endometrial lesion, MRI shows signal hyperintensity on T1-weighted imaging and signal hypointensity on T2-weighted imaging. ${ }^{9}$ However in the present case, MRI demonstrated a nodular thickened area of mild constrast enhancement showing signal hypointensity on both T1and T2-weighted imaging, possibly indicating a cancerous or fibrotic lesion. It is therefore important to have a high clinical suspicion particularly when imaging tests are negative or inconclusive, so as to prompt an operative diagnosis. Indeed reports have described preoperative confusion between endometriotic lesions and cancer, particularly in patients with lesions involving the mucosal surfaces. The presence of an ileal endometrioma may act as a mass lesion and mimic a malignancy by encroaching into the ileal lumen. ${ }^{10}$ Histopathological analysis is therefore needed to confirm the diagnosis and assess extent of spread. In our patient this showed multiple endometriotic foci and marked fibrosis evident on the serosal surface, muscularis propria and submucosa which led to stricturing of terminal ileum. Endometriosis and fibrotic changes had also resulted in appendiceal intussusception. The development of a fibrotic ileal stricture and intussusception resulted in the subocclusive episodes and intestinal obstruction.

Our patient who presented with suspected SBO was investigated with an abdominal radiograph, CT and MRI to establish the cause. Diagnosing intestinal endometriosis is challenging as gastrointestinal symptoms are nonspecific and no radiological gold standard currently exists. Laparoscopy is considered the primary diagnostic modality. ${ }^{11}$ However, imaging techniques such as ultrasonography, MRI and CT still have a role. The use of transvaginal ultrasonography is largely confined to lesions distal to the rectosigmoid junction. ${ }^{12} \mathrm{MRI}$ has been studied extensively but altered signal patterns can make it difficult to delineate histological components of an endometrioma. Biscaldi et al demonstrated the efficacy of multi-slice CT combined with water enteroclysis (MSCTe) in distinguishing endometriotic lesions from chronic inflammatory and neoplastic lesions. ${ }^{13}$ Although this technique has shown great promise it remains unsuitable for patients with obstructive symptoms.

In our patient once the endometriotic lesion was identified, definitive surgical treatment involved a right hemicolectomy, which resulted in resolution of the symptoms. Postoperatively, a referral was made to a gynaecologist for identification and treatment of endometriosis. Our case highlights an unusual presentation of endometriosis, which due to its non-specific nature may be misdiagnosed, and should therefore be considered in patients with abdominal pain and signs of bowel obstruction.

Learning points

- Endometriosis is an important cause of abdominal pain in women of reproductive age.

- Symptoms maybe non-specific and no radiological gold standard currently exists.

- Histopathology is needed to confirm the diagnosis and assess extent of spread. 


\section{Competing interests None.}

Patient consent Obtained.

\section{REFERENCES}

1. McEntee GP. Small bowel obstruction. In: Monson J, Duthie G, O'Malley K, eds. Surgical Emergencies. UK: Blackwell Science 1999:164.

2. Foster NM, McGory ML, Zingmond DS, et al. Small bowel obstruction: a population-based appraisal. J Am Coll Surg 2006;203:170-6.

3. Macafee $\mathbf{C H}$, Greer HL. Intestinal endometriosis. A report of 29 cases and a survey of the literature. J Obstet Gynaecol Br Emp 1960;67:539-55.

4. Croom RD3rdDonovan ML, Schwesinger WH. Intestinal endometriosis. Am J Surg 1984;148:660-7.

5. Yantiss RK, Clement PB, Young RH. Endometriosis of the intestinal tract: a study of 44 cases of a disease that may cause diverse challenges in clinical and pathologic evaluation. Am J Surg Pathol 2001;25:445-54.

6. Ridha JR, Cassaro S. Acute small bowel obstruction secondary to ileal endometriosis: report of a case. Surg Today 2003;33:944-7.
7. Martimbeau PW, Pratt JH, Gaffey TA. Small-bowel obstruction secondary to endometriosis. Mayo Clin Proc 1975;50:239-43.

8. Langlois NE, Park KG, Keenan RA. Mucosal changes in the large bowel with endometriosis: a possible cause of misdiagnosis of colitis? Hum Pathol 1994;25:1030-4.

9. Puglielli E, Di Cesare E, Masciocchi C. Rectal endometriosis: MRI study with rectal coil. Eur Radiol 2004;14:2362-3.

10. Melody GF. Endometriosis causing obstruction of the ileum. Obstet Gynecol 1956;8:468-72.

11. Kennedy S, Bergqvist A, Chapron C, et al.; ESHRE Special Interest Group for Endometriosis and Endometrium Guideline Development Group. ESHRE guideline for the diagnosis and treatment of endometriosis. Hum Reprod 2005;20:2698-704.

12. Gorell HA, Cyr DR, Wang KY, et al. Rectosigmoid endometriosis. Diagnosis using endovaginal sonography. J Ultrasound Med 1989;8:459-61.

13. Biscaldi E, Ferrero S, Fulcheri E, et al. Multislice CT enteroclysis in the diagnosis of bowel endometriosis. Eur Radiol 2007;17:211-19.

This pdf has been created automatically from the final edited text and images.

Copyright 2012 BMJ Publishing Group. All rights reserved. For permission to reuse any of this content visit http://group.bmj.com/group/rights-licensing/permissions.

BMJ Case Report Fellows may re-use this article for personal use and teaching without any further permission.

Please cite this article as follows (you will need to access the article online to obtain the date of publication).

Khwaja SA, Zakaria R, Carneiro HA, Khwaja HA. Endometriosis: a rare cause of small bowel obstruction.

BMJ Case Reports 2012;10.1136/bcr.03.2012.5988, Published XXX

Become a Fellow of BMJ Case Reports today and you can:

- Submit as many cases as you like

- Enjoy fast sympathetic peer review and rapid publication of accepted articles

- Access all the published articles

- Re-use any of the published material for personal use and teaching without further permission

For information on Institutional Fellowships contact consortiasales@bmigroup.com

Visit casereports.bmj.com for more articles like this and to become a Fellow

Keep up to date with all published cases by signing up for an alert (all we need is your email address) http://casereports.bmj.com/cgi/alerts/etoc 